\title{
$X$-ray investigation of nanostructured stain-etched porous silicon
}

\author{
P. G. Abramof, ${ }^{\text {a) }}$ A. F. Beloto, A. Y. Ueta, and N. G. Ferreira ${ }^{\text {) }}$ \\ Laboratório Associado de Sensores e Materiais (LAS), Instituto Nacional de Pesquisas Espaciais (INPE), \\ CP 515, 12245-970 São José dos Campos, Sao Paulo, Brazil
}

(Received 19 August 2005; accepted 29 November 2005; published online 20 January 2006)

\begin{abstract}
The structure of porous silicon layers was accurately investigated by diverse x-ray methods. A series of samples with etching times varying from 1 to 10 min was produced by chemical etch using a $\mathrm{HF} / \mathrm{HNO}_{3}$-based solution assisted with $\mathrm{NaNO}_{2}$. The porosity determined from low-angle x-ray reflectivity spectra was found to fluctuate from $35 \%$ to $55 \%$ as the etch proceeds. Reciprocal space mapping around the (004) Si lattice point revealed that the Si crystallites are deformed due to a distribution of in-plane compressive strain caused by the neighboring pores, which leads to an expansion of the perpendicular lattice parameter. No signature of mosaicity was found. The perpendicular strain could be precisely determined by fitting the x-ray-diffraction spectra, measured in the triple-axis configuration, to a set of Voigt and Gaussian distributions. These strain distributions are certainly associated with the different population of crystallite sizes formed during the stain etching process. We were able here to determine the temporal evolution of the strain inside the nanostructured porous silicon. (C) 2006 American Institute of Physics.
\end{abstract}

[DOI: 10.1063/1.2162273]

\section{INTRODUCTION}

Porous silicon (PS) has been widely studied as an interesting material with potential for many device applications, such as optical components, gas sensors, and biotechnology. Since the discovery of PS room-temperature luminescence, ${ }^{1}$ many studies have been performed in order to understand its formation process and properties. ${ }^{2-5}$ Electrochemical etching as well as stain etching processes are usually used for producing PS on monocrystalline silicon. By stain etching, a solution containing $\mathrm{HF}, \mathrm{HNO}_{3}$, and water is used in a process where an oxidation of silicon atoms occurs by hole injection from nitric acid and, simultaneously, its reduction produces $\mathrm{NO}$ and water. The stain etching can be an advantageous method in the formation of porous silicon layers, ${ }^{6-8}$ mainly for substrates with higher doping level.

Promising applications are related to the photoluminescence of micro and nanostructured porous Si. The origin of this phenomenon is mainly attributed to quantum confinement effects in nanometer size silicon crystallites. ${ }^{9-12}$ Raman-scattering spectroscopy, together with the phonon confinement model, has been demonstrated as a powerful technique to investigate PS structure. ${ }^{13-15}$ Furthermore, highresolution $\mathrm{x}$-ray techniques have been applied to obtain information about the peculiar structure and strain of porous $\mathrm{Si}^{16-19}$ However, the determination of size and shape distribution of crystallites in PS layers is still a subject of intense interest.

This article reports on an investigation of porous silicon layer structure using several $\mathrm{x}$-ray techniques. The samples were produced by stain etching with etching times varying from 1 to $10 \mathrm{~min}$. The porosity, determined by grazing inci-

\footnotetext{
${ }^{a}$ Permanent address: Centro de Desenvolvimento de Tecnologia e Recursos Humanos (CDT), 12242-800 São José dos campos, Sao Paulo, Brazil.

b) Author to whom correspondence should be addressed; electronic mail: neidenei@las.inpe.br
}

dence x-ray reflectivity, exhibited an oscillatory behavior as a function of etching time. Rocking curves, which are $\omega$ scans with the open detector fixed at the respective $2 \Theta$ angle, were measured around the (004) Si Bragg reflection. Besides the bulk Si peak, a diffraction band relative to the porous material was observed at the lower-angle side. In order to distinguish changes in orientation of the lattice planes from variations in the lattice spacing, reciprocal space mapping around the (004) lattice point was performed. Subsequently, we have carefully measured $\omega / 2 \Theta$ scans around the (004) $\mathrm{Si}$ Bragg peak in the triple-axis configuration with a longer integration time to increase accuracy. The perpendicular strain inside the crystallites that from the porous layer was determined from a best-fit procedure using a set of Voigt and Gaussian distributions. The results from this $\mathrm{x}$-ray analysis asserted the stain etching as a competitive process with different population of $\mathrm{Si}$ crystallite sizes associated with each etching stage.

\section{EXPERIMENT}

Porous silicon layers were produced by chemical (stain) etching on (001) silicon wafers of $15 \times 15 \mathrm{~mm}^{2}$, boron doped ( $p^{+}$-type) corresponding to a resistivity of $0.01-0.02 \Omega \mathrm{cm}$. A solution composed of $\mathrm{HF}$ and $\mathrm{HNO}_{3}$ in a volume ratio of 500:1 was used to etch the samples. ${ }^{8}$ The Si wafers were etched in a disk of $11 \mathrm{~mm}$ in diameter. Since the etching is very sensitive to the type and doping concentration of the silicon wafer, ${ }^{20}$ a small amount of $\mathrm{NaNO}_{2}(0.1 \mathrm{~g} / 1)$ was added to the etch solution in order to homogenize the stain etching, according to the procedure described by Kelly et al. ${ }^{7}$ A series of PS samples with etching times ranging from 1 to $10 \mathrm{~min}$ was produced. The porous Si layers were $1-4 \mu \mathrm{m}$ thick as evaluated from scanning electron microscopy.

The samples were characterized by X-ray reflectivity and diffraction measured in a Philips X'Pert MRD high- 
resolution x-ray diffractometer. The grazing incidence $\mathrm{x}$-ray reflectivity measurements were performed with the apparatus in the following configuration: $x$-ray tube in line focus; Soller slit, $1 / 32^{\circ}$ divergence slit and an attenuator in the primary optics; parallel beam collimator, $0.1 \mathrm{~mm}$ antiscatter and flat crystal graphite monochromator as secondary optics. Before starting measuring, the sample height and the goniometer zero points $(2 \theta=\omega=0)$ are precisely adjusted. The attenuator is used for angles lower than the critical value, where total reflection occurs, to avoid the detector damage due to a very intense incident radiation. The attenuator factor is automatically computed, allowing a dynamical scale of eight orders of magnitude during continuous measurement. For the diffraction measurements, the $\mathrm{Cu} \mathrm{x}$-ray tube of the diffractometer is changed to point focus. A four-crystal Ge (220) monochromator is placed in the primary optics, leading to an axial divergence of 12 arc sec for the incident beam. There are two possible configurations for the diffracted beam. It may go directly to an open detector with an aperture of $1^{\circ}$ (rocking curve configuration), or may pass through a crystal analyzer placed between the sample and detector, which reduces the acceptance angle of the detector to 12 arc sec (triple-axis configuration).

\section{RESULTS AND DISCUSSION}

The grazing incidence $x$-ray reflectivity profiles of all the PS samples were recorded with an $\omega / 2 \theta$ scan from $\omega$ $=0.1^{\circ}$ to $2^{\circ}$. The upper panel of Fig. 1 shows the reflectivity spectra in a logarithmic scale for the porous Si samples with 1,5 , and 8 min of etching times. In $\mathrm{x}$-ray grazing incidence geometry, the beam undergoes a total external reflection for incidence angles smaller than the critical angle $\left(\omega_{c}\right)$ due to a refractive index whose real part is slightly smaller than unity. For angular values larger than the critical angle, the beam penetrates the material. The presence of pores and crystallites in PS layers leads to a diffuse scattering of the incidence beam, which determines the decrease of the specular reflectivity for angles larger than $\omega_{c}$, and hence no interference fringes could be observed for these samples. The critical angle for total reflection is given by $\omega_{c}=\sqrt{2} \delta$, where $\delta$ is related to the electron density of the medium. It is the angle value at which the intensity of the reflectivity curve falls to its half. ${ }^{21}$ The lower panel of Fig. 1 plots the reflectivity curves of the PS samples in a linear scale from $0^{\circ}$ to $0.36^{\circ}$ along with the spectrum of a bulk silicon, for comparison. We have obtained from this graph the value $\omega_{c}=0.228^{\circ}$ for bulk $\mathrm{Si}$, close to the theoretical value $\omega_{c}=0.223^{\circ}$. Critical angles for the PS samples with etching times of 1,5 , and 8 min were $\omega_{c}=0.184^{\circ}, 0.154^{\circ}$, and $0.160^{\circ}$, respectively. Note that the critical angles of the PS layers are always smaller than the one for bulk Si due to their lower density.

The critical angles determined from the x-ray reflectivity profiles are used to determine the porosity of the PS layers. This important property of the porous material can be determined by the following relation: ${ }^{18}$
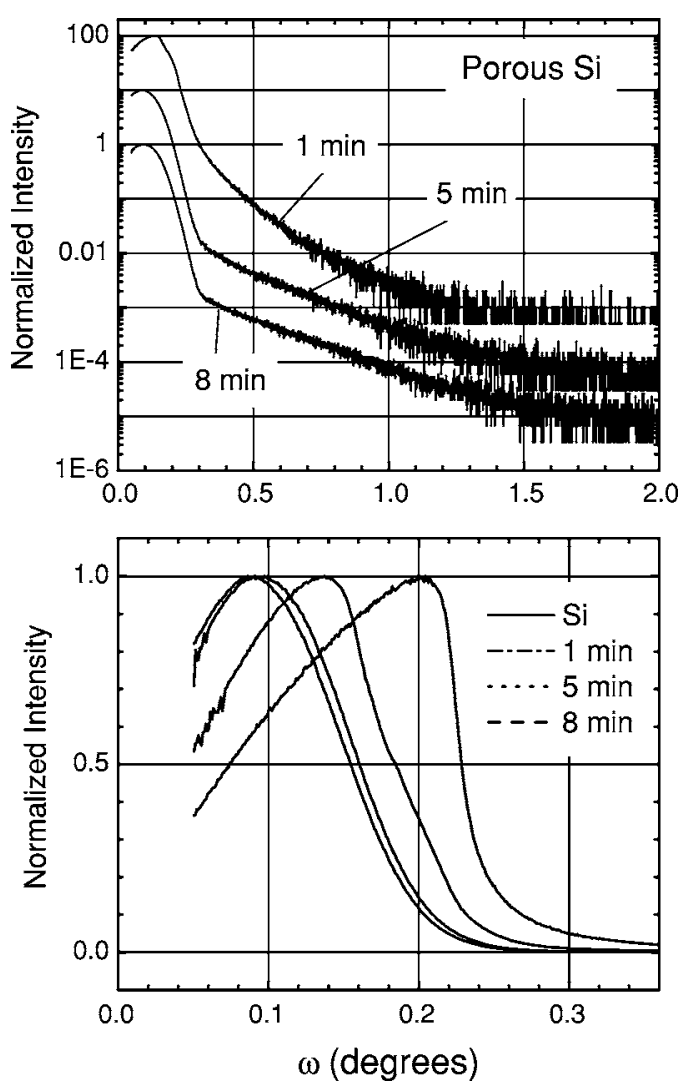

FIG. 1. Grazing incidence x-ray reflectivity profiles of porous Si samples with 1,5 , and $8 \mathrm{~min}$ of etching time. The upper panel shows the whole spectra in a logarithmic scale and shifted to help visualization. The lower panel plots the reflectivity curves in a linear intensity scale from $0^{\circ}$ to $0.36^{\circ}$ together with the spectrum of bare silicon.

$$
P=1-\left(\frac{\omega_{c}^{\mathrm{PS}}}{\omega_{c}^{\mathrm{Si}}}\right)^{2}
$$

where $\omega_{c}^{\mathrm{PS}}$ layer and $\omega_{c}^{\mathrm{Si}}$ are the critical angles in radians of the PS layer and of bulk silicon, respectively.

The porosity of all the PS samples was calculated and the results are displayed in Fig. 2. It does not show a monotonic behavior with etching time, but it oscillates. An increase from $35 \%$ to $48 \%$ is observed, as the etching time

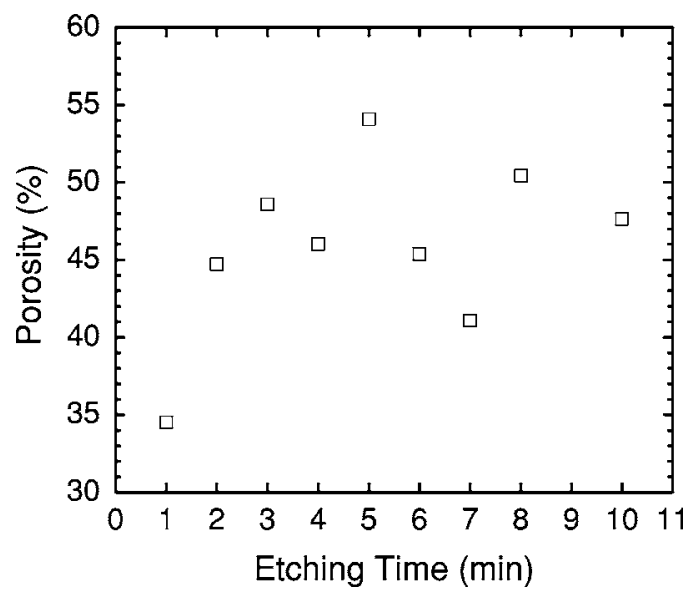

FIG. 2. Porosity, as obtained from x-ray reflectivity, for stain etchinged porous $\mathrm{Si}$ as a function of etching time. The experimental error is within the square size. 


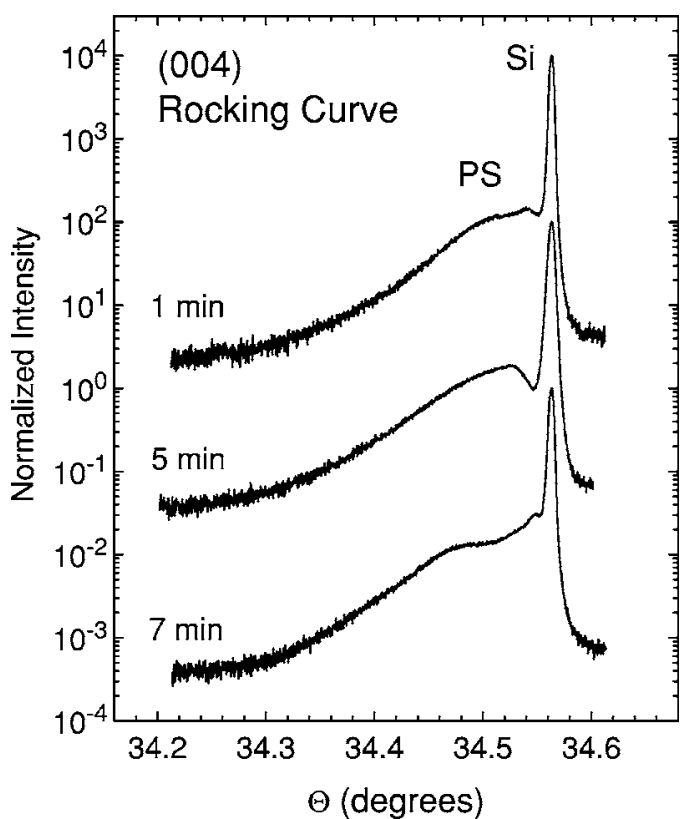

FIG. 3. X-ray rocking curves measured around the $\mathrm{Si}$ (004) Bragg peak for porous $\mathrm{Si}$ samples with 1,5 , and $7 \mathrm{~min}$ of stain etching. The spectra are shifted in the ordinate for a better visualization.

increases from 1 to $3 \mathrm{~min}$ and the porosity oscillates in the range from $40 \%$ to $55 \%$ for etching times ranging from 3 to $10 \mathrm{~min}$. This behavior may be related to the formation of the PS layer as the etch proceeds. It is important to notice that $\mathrm{x}$-ray reflectivity measurement probes only a depth of 250 $\mathrm{nm}$ from the surface, as estimated from the attenuation length of the $\mathrm{Cu} K \alpha$ radiation incident at an angle of $0.3^{\circ}$ in the Si surface. In this sense, it probably probes the porosity change in this depth due to a nonuniform PS thickness formed by nanostructure and islandlike surface morphology. This result indicates that the stain etching is a continuous process, and the porous Si surface is submitted to a competitive etch as the etching time increases. ${ }^{22}$

In order to measure the x-ray-diffraction curves of the PS samples, the diffractometer was firstly changed to the rocking curve configuration. The rocking curves (RC's), which are $\omega$ scans measured with the open detector fixed at the desired $2 \Theta$ angle, were measured around the (004) $\mathrm{Si}$ Bragg diffraction peak of all the PS samples. Figure 3 shows the (004) RC's for the PS samples with etching times of 1, 5, and $7 \mathrm{~min}$. The spectra exhibit an intense and narrow diffraction peak corresponding to the bulk $\mathrm{Si}$ substrate, and, at lower angles, a less intense band derived from the diffraction in the $\mathrm{Si}$ crystallites of the porous material. This band extends down to $0.3^{\circ}$ relative to the bulk Si peak position and seems to have a structure composed of different peaks. For example, in the sample with $7 \mathrm{~min}$ of etching time, we can identify even two peaks with different widths and intensities in this diffraction band. The scattered intensity is observed only for angles lower than the Si substrate peak, which indicates that the lattice of the crystallites that compose the porous $\mathrm{Si}$ is distorted with an in-plane compressive strain that leads to an expansion of the perpendicular lattice parameter. Since the x-ray diffraction in the porous material appears as a band, we may infer that there is a strain distribution inside the crystallites, which depends on their size, instead of a homogeneous strain. It is also important to comment that the rocking curves displayed here for the stain etched porous $\mathrm{Si}$ samples showed a different form than the ones measured for anodic porous $\mathrm{Si}$ obtained by the standard electrochemical process. In the latter case, the RC's exhibit two narrow diffraction peaks (one of the Si substrate and another at lower angles due to the porous Si layer) over a broad hump attributed to diffuse scattering. ${ }^{17}$ This result indicates that there are significant structural differences between stain etched and anodic-etched porous Si materials. At this point, we are able to affirm that there is a lot of information about the PS structure inside the $\mathrm{x}$-ray rocking curve. Unfortunately, since the $\mathrm{RC}$ 's are measured with an open detector, it is not possible to distinguish changes in orientation of the lattice planes (mosaicity) from variations in the lattice spacing $(\Delta d / d)$.

In order to resolve these differences, it is necessary to measure the so-called reciprocal space map (RSM), using the diffractometer in the triple-axis configuration. The RSM of a specific reciprocal lattice point is obtained by measuring several $\omega / 2 \theta$ scans with different $\omega$ offsets. The map is usually plotted in reciprocal space coordinates $Q_{x}$ and $Q_{z}$ that can be obtained from the Bragg angle $\Theta$ and the angle $\omega$, measured between the incident beam and the sample surface, using the following relations:

$$
Q_{x}=-\frac{2}{\lambda} \sin (\omega-\Theta) \sin \Theta, \quad Q_{z}=\frac{2}{\lambda} \cos (\omega-\Theta) \sin \Theta,
$$

where $\lambda$ is the incident $\mathrm{x}$-ray wavelength, in this case $\lambda$ of $\mathrm{Cu} K \alpha_{1}$ radiation. We measured the reciprocal space map of the diffracted x-ray intensity around the symmetric (004) lattice point of the PS samples. Figure 4 shows the twodimensional map for the PS sample etched during $7 \mathrm{~min}$, with isointensity contour lines plotted in $Q_{x}$ along the [110] in-plane azimuth (parallel to the sample surface) and $Q_{z}$ along the [001] direction (perpendicular to the sample surface). It is important to point out that, in RSM's, the scattered intensity due to changes in lattice spacing is observed along the $Q_{z}$ direction, while mosaicity produces a broadening in the scattered x-ray intensity in the $Q_{x}$ direction. Besides the bulk Si Bragg diffraction peak observed at $Q_{x}=0$ and $Q_{z}=0.7367 \AA^{-1}$, the RSM exhibits scattered x-ray intensity down to $Q_{z}=0.7300 \AA^{-1}$ due to the porous $\mathrm{Si}$ layer. Note that the map extends over a range of $6 \times 10^{-3} \AA^{-1}$ along the $Q_{z}$ axis, while along the $Q_{x}$ axis it lies only in a range of 1 $\times 10^{-4} \AA^{-1}$. Therefore, all x-ray intensity is almost scattered along the $Q_{z}$ direction, with only a small broadening in the $Q_{x}$ direction. This fact is clearly seen in the threedimensional surface of the (004) RSM shown in the upper part of Fig. 4. The diffuse scattering observed by Buttard et $a l .{ }^{17}$ for porous Si produced by anodic etching appears in the (004) RSM as a narrow streak along $Q_{x}$ or as isotropic scattered intensity, depending on the morphology of the porous layer. Therefore, we can conclude that no significant diffuse scattering takes place during the diffraction measurements on our stain etched porous Si samples. Furthermore, the RSM analysis confirms that the crystallites of the porous $\mathrm{Si}$ are under a distribution of in-plane compressive strain, which leads to an increase of the perpendicular lattice parameter. 


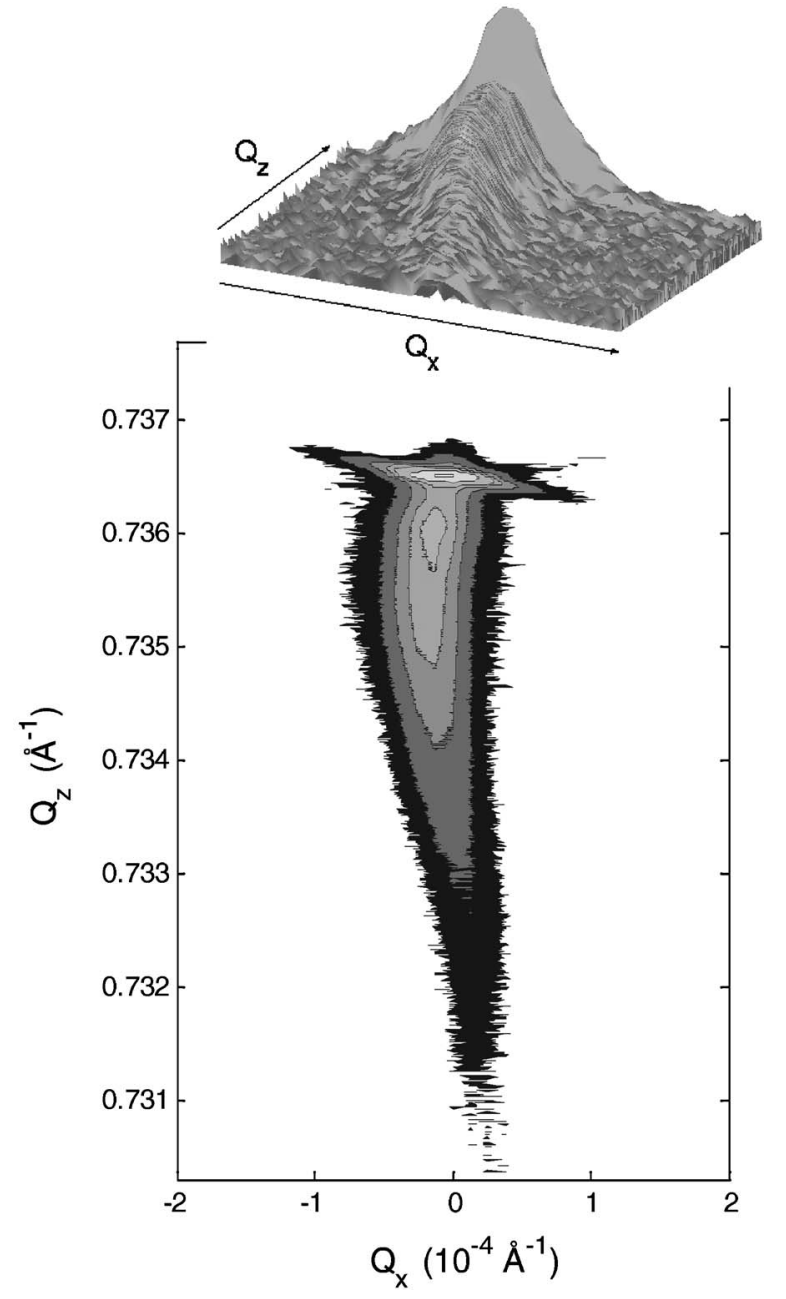

FIG. 4. Reciprocal space map measured around the (004) Si lattice point and plotted in reciprocal coordinate $Q_{x}$ parallel to the [110] in-plane azimuth and $Q_{z}$ along the [001] direction. The isointensity contour lines are at $80000,8000,1200,600,200,40$, and 8 counts/s. The three-dimensional view of the log-transformed intensities is shown in the upper part. Note that the scattered intensity lies practically along the $Q_{z}$ direction.

The distortion in the crystalline lattice of the porous $\mathrm{Si}$ is basically in the lattice parameter, presenting almost no signature of mosaicity.

Structural parameters of such porous material may be analyzed and evaluated from a more accurate $\mathrm{x}$-ray method. We have carefully measured $\omega / 2 \Theta$ scans around the (004) $\mathrm{Si}$ Bragg reflection in the triple-axis configuration, due to its higher resolution. This measurement corresponds to a scan along the $Q_{z}$ direction at $Q_{x}=0$ in the RSM of Fig. 4, made with a longer integration time to increase the accuracy. Figure 5 shows the $\omega / 2 \theta$ scan measured in the triple-axis configuration for the PS samples with etching times of 1,5 , and 7 min. Triple-axis spectra have shown a more resolved structure of the diffraction band relative to porous material than the corresponding rocking curves of Fig. 3. It is evident that a relative narrow peak is close to the Si bulk peak, and one extra broader peak shows the diffraction band. In order to reproduce the x-ray-diffraction curve measured in the tripleaxis configuration, it has been fitted with a combination of different distributions. The best distribution combination chosen was composed of two Voigt and two Gaussian curves.

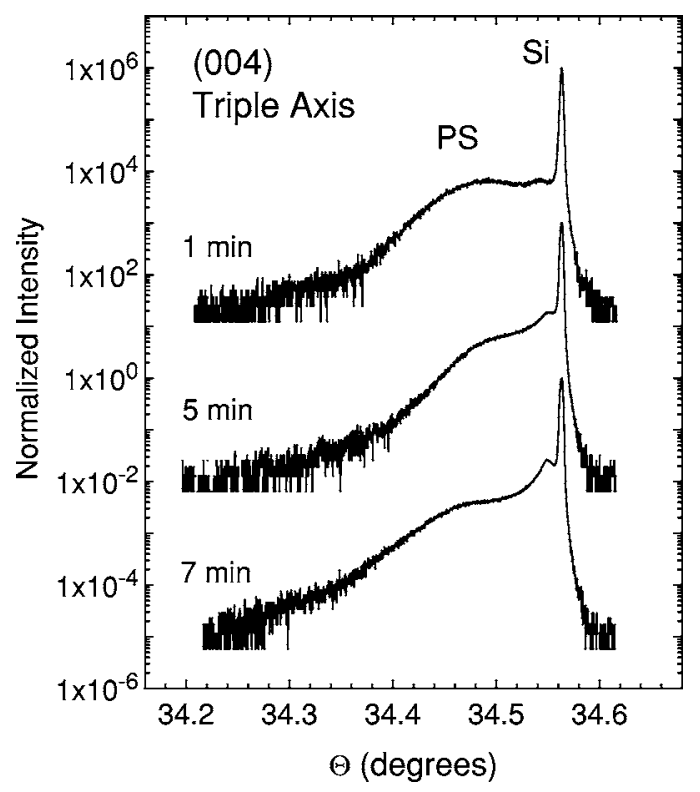

FIG. 5. $\omega / 2 \theta$ scan measured in the triple-axis configuration around the (004) diffraction peak for porous Si samples with different etching times. For clarity, the spectra are shifted in the ordinate.

Figure 6 shows the best fit (solid line) to the measured $x$-ray spectrum (circles) of the PS sample with $7 \mathrm{~min}$ of etching time. We named the peaks that compose the diffraction band of the PS layer as PS1, PS2, and PS3. The Voigt curves of the Si for PS1 peaks and Gaussian distributions for PS2 and PS3, found to reach the best fit, are also shown in Fig. 6 . This result indicates that there are at least three preferential distributions of crystallite sizes inside the PS layer. Peak PS1 corresponds to crystallites with larger dimensions, which are under less strain and could be fitted with a Voigt distribution similar to the bulk Si. The other Gaussian distributions are related to smaller crystallites which are more stressed.

In order to quantify the strain inside the porous Si layer, this fitting process was applied to all the PS samples. The perpendicular strain relative to each distribution (PS1, PS2, and PS3) can be obtained using the following relation:

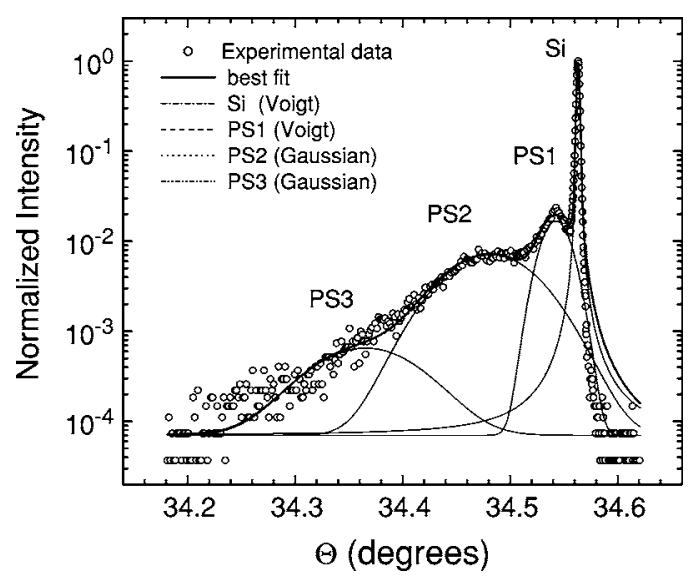

FIG. 6. Best fit to the X-ray spectrum of Fig. 5 for the porous Si sample with 7 min of etching time. The Voigt and Gaussian curves used to reach the best fit are also shown. 


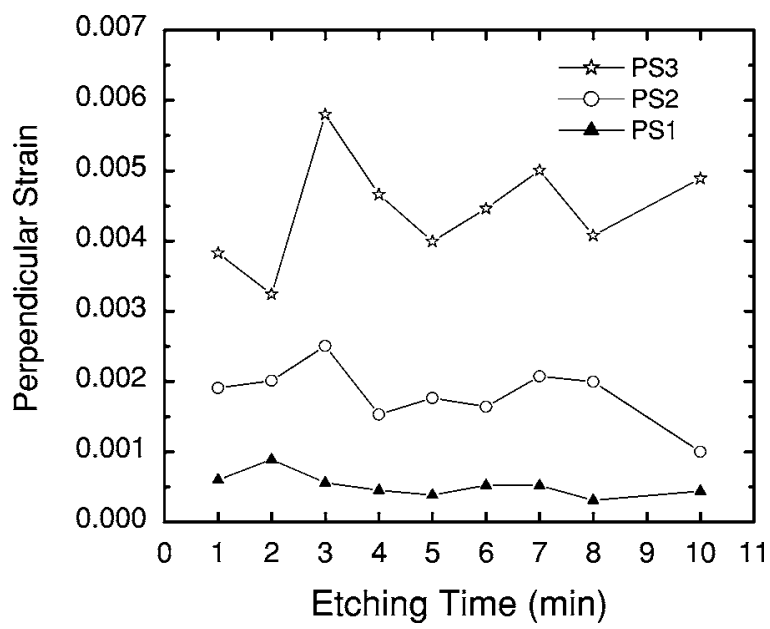

FIG. 7. Perpendicular strain for the PS1, PS2, and PS3 peaks as a function of etching time.

$$
\varepsilon_{\perp}=-\frac{\Delta \Theta}{\tan \Theta_{\mathrm{Si}}},
$$

where $\Delta \Theta$ is the angular difference between the central position of each peak and the bulk Si peak position $\Theta_{\mathrm{Si}}$. Figure 7 shows the perpendicular strain for the PS1, PS2, and PS3 distributions as a function of etching time. Note that the strain for the PS1 peak is in the order of $10^{-4}$, while it is in the order of $10^{-3}$ for PS2 and PS3.

This behavior is related to the porous $\mathrm{Si}$ formation specifically using chemical etching. In a different way from electrochemical PS formation, Winton et al..$^{22}$ have discussed the stain etch as a localized formation of nanostructured porous by a propagation of a discrete reaction interface into the silicon substrate. Indeed, there is a competitive process between the nanostructures, responsible for the photoluminescence, and islandlike structures formed as the etch proceeds. Silicon agglomerates or islandlike structures with different sizes and shapes always appear in the surface and in the PS layer depth. Consequentially, different crystallite distributions may be associated with each etch stage. The three strain distributions determined form the x-ray-diffraction analysis here are clearly associated with the different population of crystallite sizes formed during the stain etching process. It is important to emphasize that the (004) x-ray-diffraction profile measured in the triple-axis configuration really probes the perpendicular strain inside the crystallites that compose the porous Si layers. In this sense, they are much more resolute than the rocking curves and can be used to extract quantitative information about the PS structure.

\section{CONCLUSIONS}

Different X-ray techniques were applied to investigate the structure of stain etched porous Si layers as a function of etching time. Grazing incidence x-ray reflectivity was measured to determine the porosity, which fluctuated between $35 \%$ and $55 \%$ as the etching time varied from 1 to $10 \mathrm{~min}$.
The diffraction band, observed at the lower-angle side of the (004) x-ray rocking curves, indicated that there is an inhomogeneous strain distribution inside the crystallites of the porous material. Reciprocal space mapping around the (004) lattice point revealed that the distortion caused by the pores in the $\mathrm{Si}$ crystalline lattice is practically in lattice spacing with no signature of mosaicity. The results of the RSM analysis demonstrated that the crystallites of the porous $\mathrm{Si}$ are under a distribution of in-plane compressive strain, leading to a respective expansion of the perpendicular lattice parameter. The perpendicular strain was accurately determined by fitting the (004) $\omega / 2 \theta$ scans, carefully measured in the triple-axis configuration, with a composition of Voigt and Gaussian distributions. These distributions were found to be clearly associated with the different population of crystallite sizes, which varies during the stain etching process. Due to the accuracy and resolution of the x-ray-diffraction profile measured in the triple-axis configuration, it was possible to determine here the temporal evolution of the strain inside the stain etched Si crystallites.

\section{ACKNOWLEDGMENTS}

The authors are very grateful to FAPESP (Process No. 02/10604-6) and CNPq for the financial support.

${ }^{1}$ L. T. Canham, Appl. Phys. Lett. 57, 1046 (1990).

${ }^{2}$ In Porous Silicon and Technology, edited by J. C. Vial and J. Derrien (Springer, Berlin, 1994).

${ }^{3}$ In Structural and Optical Properties of Porous Silicon Nanostructures, edited by G. Amato, C. Delerue, and H. J. von Bardeleben (Gordon and Breach, Amsterdam, 1997).

${ }^{4}$ A. G. Cullis, L. T. Canhan, and P. D. J. Calcott, J. Appl. Phys. 82, 909 (1997).

${ }^{5}$ In Electrochemistry of Silicon, edited by V. Kehman (Wiley-VCH, 2002). ${ }^{6}$ S. Shih, K. H. Jug, T. H. Y. Hsieh, J. Sarathy, J. C. Campbell, and D. L. Kwong, Appl. Phys. Lett. 60, 15 (1992).

${ }^{7}$ M. T. Kelly, J. K. M. Chun, and A. B. Bocarsly, Appl. Phys. Lett. 64, 1693 (1994).

${ }^{8}$ É. Vazsonyi, E. Szilágyi, P. Petrik, Z. E. Horváth, T. Lohner, M. Fried, and G. Jalsovszky, Thin Solid Films 388, 295 (2001).

${ }^{9}$ H. Richter, Z. P. Wang, and L. Ley, Solid State Commun. 39, 625 (1981).

${ }^{10}$ I. H. Campbel and P. M. Fauchet, Solid State Commun. 58, 739 (1986).

${ }^{11}$ Z. Sui, P. P. Leong, I. P. Herman, G. S. Higashi, and H. Temkin, Appl. Phys. Lett. 60, 2086 (1992).

${ }^{12}$ M. Yang, D. Huang, P. Hao, F. Zhang, X. Hou, and X. Wang, J. Appl. Phys. 75, 651 (1994).

${ }^{13}$ D. Papadimitriou, J. Bitsakis, J. M. López-Villegas, J. Samitier, and J. R. Morante, Thin Solid Films 349, 293 (1999).

${ }^{14}$ S. Manotas, F. Agulló-Rueda, J. D. Moreno, F. Ben-Hander, R. GuerreroLemus, and J. M. Martínez-Duart, Phys. Status Solidi A 182, 245 (2000).

${ }^{15}$ P. G. Abramof, N. G. Ferreira, A. F. Beloto, and A. Y. Ueta, J. Non-Cryst. Solids 338-340, 139 (2004).

${ }^{16}$ D. Bellet and G. Dolino, Thin Solid Films 276, 1 (1996).

${ }^{17}$ D. Buttard, D. Bellet, and G. Dolino, J. Appl. Phys. 79, 8060 (1996).

${ }^{18}$ D. Buttard, G. Dolino, D. Bellet, T. Baumbach, and F. Rieutord, Solid State Commun. 109, 1 (1999).

${ }^{19}$ V. Chamard, S. Setzu, and R. Romestain, Appl. Surf. Sci. 191, 319 (2002).

${ }^{20}$ A. J. Steckl, J. Xu, H. C. Mongul, and S. Mogren, Appl. Phys. Lett. 62, 1982 (1993)

${ }^{21}$ L. G. Parrat, Phys. Rev. 95, 359 (1954).

${ }^{22}$ M. J. Winton, S. D. Russell, and R. Gronsky, J. Appl. Phys. 82, 1 (1997). 